Proceedings of the Edinburgh Mathematical Society (2004) 47, 533-552 (C)

DOI:10.1017/S0013091504000197 Printed in the United Kingdom

\title{
TRANSFORMATION OF STURM-LIOUVILLE PROBLEMS WITH DECREASING AFFINE BOUNDARY CONDITIONS
}

\author{
PAUL A. BINDING ${ }^{1}$, PATRICK J. BROWNE ${ }^{2}$, WARREN J. CODE ${ }^{2}$ \\ AND BRUCE A. WATSON ${ }^{3}$ \\ ${ }^{1}$ Department of Mathematics and Statistics, University of Calgary, \\ Calgary, Alberta T2N 1N4, Canada (binding@ucalgary.ca) \\ ${ }^{2}$ Mathematical Sciences Group, Department of Computer Science, University of \\ Saskatchewan, Saskatoon, Saskatchewan S7N 5E6, Canada \\ ${ }^{3}$ Department of Mathematics, University of the Witwatersrand, \\ Private Bag 3, PO WITS 2050, South Africa
}

(Received 21 January 2004)

\begin{abstract}
We consider Sturm-Liouville boundary-value problems on the interval $[0,1]$ of the form $-y^{\prime \prime}+q y=\lambda y$ with boundary conditions $y^{\prime}(0) \sin \alpha=y(0) \cos \alpha$ and $y^{\prime}(1)=(a \lambda+b) y(1)$, where $a<0$. We show that via multiple Crum-Darboux transformations, this boundary-value problem can be transformed 'almost' isospectrally to a boundary-value problem of the same form, but with the boundary condition at $x=1$ replaced by $y^{\prime}(1) \sin \beta=y(1) \cos \beta$, for some $\beta$.
\end{abstract}

Keywords: Sturm-Liouville; eigenparameter-dependent boundary conditions; Darboux transformation 2000 Mathematics subject classification: Primary 34B07; 47E05; 34L05

\section{Introduction}

Our aim is to transform, 'almost' isospectrally, a Sturm-Liouville equation

$$
-y^{\prime \prime}(x)+q(x) y(x)=\lambda y(x), \quad 0 \leqslant x \leqslant 1,
$$

with boundary conditions

$$
\begin{aligned}
& Y(0)=\cot \alpha, \quad 0 \leqslant \alpha<\pi, \\
& Y(1)=a \lambda+b
\end{aligned}
$$

where $a<0$, into a 'standard' Sturm-Liouville problem. By 'almost' we mean that at most two eigenvalues will change, and by 'standard' we mean a problem where the differential equation is regular and the boundary conditions are independent of $\lambda$. We shall consistently use upper-case Roman letters to denote logarithmic derivatives, so $Y$ means $y^{\prime} / y$ in (1.2), (1.3). We assume that $q$ is real and integrable on $[0,1]$, and if $\alpha=0$, then (1.2) is interpreted as $y(0)=0$. The decision to keep (1.2) independent of $\lambda$ is for 
simplicity of presentation; cf. [5] for analogous questions with both boundary conditions $\lambda$ dependent.

Sturm-Liouville problems with $\lambda$-dependent boundary conditions of the form

$$
Y(1)=f(\lambda)
$$

have been studied a good deal from the viewpoints of both theory and applications. Most applications are to affine conditions like (1.3); [10] and [18] have extensive reference lists, but see, for example, $[\mathbf{3}]$ for square-root dependence and also $[\mathbf{1 3}]$ for the bilinear case

$$
f(\lambda)=\frac{a \lambda+b}{c \lambda+d}, \quad\left|\begin{array}{ll}
a & b \\
c & d
\end{array}\right|>0 .
$$

Theoretical investigations involving Herglotz-Nevanlinna functions $f$ can be found in [17], rational $f$ in, for example, [16], and a combination of these properties was considered in [7]. More general $\lambda$ dependence, where $f$ is a ratio of holomorphic functions, was studied in, for example, [12]. We hope to use the material here as a foundation for treating some of the above cases, and also in the study of inverse spectral problems (cf. [8]).

The transformation we seek was carried out for the case $a>0$ in $[\mathbf{6}]$, and we now briefly describe some of the ideas involved for the simplest (non-Dirichlet) case, $\alpha>0$. We start with a 'base function' $z$, i.e. a non-vanishing solution of (1.1) for some fixed $\lambda$. Then $Z\left(=z^{\prime} / z\right)$ can be used to transform $y$ to $\hat{y}=y^{\prime}-Z y$ and $q$ to $\hat{q}=q-2 Z^{\prime}$ in (1.1). Equivalent expressions were given by Darboux $[\mathbf{1 4}$, p. 132] and we shall call this a Darboux transformation. Darboux did not consider boundary conditions, but if we require $z$ to obey (1.2), (1.3), then $\hat{y}$ satisfies boundary conditions independent of $\lambda$.

In [6], $z$ was chosen as an eigenfunction of (1.1)-(1.3), and to be sure that an eigenfunction of one sign exists, one needs some oscillation theory, which is conveniently carried out via the Prüfer angle $\theta$. Indeed the eigenvalues $\lambda_{0}, \lambda_{1}, \ldots$ are given by the abscissae at the intersections of the line (1.3) with the graph of

$$
Y(1)=\cot \theta(1, \lambda)
$$

The latter has countably many branches $\mathcal{B}_{0}, \mathcal{B}_{1}, \ldots$, where points on $\mathcal{B}_{k}$ correspond to solutions $y$ of $(1.1),(1.2)$ with $k$ zeros on $(0,1)$. Since (1.3) (for $a>0$ ) intersects $\mathcal{B}_{0}$ (and indeed each $\mathcal{B}_{k}$ ) precisely once, an eigenfunction $z$ exists as required.

In our case $a<0$, however, (1.3) need not intersect $\mathcal{B}_{0}$, and even if it does so, there will be two intersections (counted algebraically). Roughly, one net 'extra' eigenvalue has been introduced, compared with the case $a>0$. When (1.3) does not intersect $\mathcal{B}_{0}$, the 'extra' eigenvalue is paired with the missing one from $\mathcal{B}_{0}$ to give either two extra eigenvalues (counted algebraically) on some further branch $\mathcal{B}_{k}$, or else one non-real conjugate pair. The various possibilities (and their connections with algebraic multiplicities of eigenvalues) are analysed in $\S 2$.

Continuing with the case $a<0<\alpha$, we find that a Darboux transformation still reduces (1.1)-(1.3) to a 'standard' problem if (1.3) intersects $\mathcal{B}_{0}$. This case, where the 
'extra' eigenvalue is 'removed' from $\mathcal{B}_{0}$, will be detailed in $\S 4$. In the case where all eigenvalues are real, it turns out that an extension of Darboux's transformation involving two base functions is needed to remove the extra eigenvalue from a further branch $\mathcal{B}_{k}$ for $k>0$. This contrasts with the case of (1.4), where a single base function suffices for such removal [6].

A Darboux-type transformation with multiple base functions (whose 'modified' Wronskian, see Definition 3.1, replaces $z$ used previously) was described by Crum [11], and we shall refer to our version noted above as a (double) Crum transformation. (The names of Darboux and/or Crum are associated with such transformations in much of the literature.) Actually, Crum used the first $n$ eigenfunctions for his base functions, corresponding to $n$ iterated Darboux (whom he did not reference) transformations. Our version is closer to that of Adler, who allowed two eigenfunctions with oscillation counts differing by one [1, Lemma 1], but both Crum and Adler produced singular transformed problems. To achieve regularity, we instead use two base functions which need not be eigenfunctions, but have the same oscillation count. The background for all the transformations we need (which for non-real eigenvalues use up to four base functions) is presented in $\S 3$.

The Dirichlet case $\alpha=0$ is more complicated than $\alpha>0$, and for example a double Crum transformation may be needed even when (1.3) intersects $\mathcal{B}_{0}$, while two such transformations in tandem are required when (1.1)-(1.3) has a triple eigenvalue. All cases requiring double Crum transformations are covered in $\S 5$. Finally, non-real eigenvalues of (1.1)-(1.3) are treated in $\S 6$. When $\alpha>0$, a triple Crum transformation produces a standard problem, but when $\alpha=0$, one needs a quadruple Crum transformation followed by a single one (i.e. of Darboux type).

\section{Preliminaries}

We shall rely on Prüfer theory associated with (1.1), (1.2). If $y(x, \lambda)$ is a solution of (1.1) and (1.2), then we put

$$
y=\rho \sin \theta, \quad y^{\prime}=\rho \cos \theta,
$$

where $\theta$ is the Prüfer angle associated with (1.1) and (1.2). Differentiating, we see that $\theta$ obeys the first-order initial-value problem

$$
\theta^{\prime}=\cos ^{2} \theta+(\lambda-q) \sin ^{2} \theta, \quad \theta(0, \lambda)=\alpha .
$$

Atkinson [2] provides a comprehensive account of this theory but it suffices here for us to note that $\theta(1, \lambda)$ is increasing in $\lambda, \theta(x, \lambda) \rightarrow 0$ as $\lambda \rightarrow-\infty$, and $\theta(x, \lambda) \rightarrow \infty$ as $\lambda \rightarrow \infty$ for each $x \in(0,1]$. The graph of $\cot \theta(1, \lambda)$ has branches $\mathcal{B}_{0}, \mathcal{B}_{1}, \ldots$ corresponding to $\lambda$ intervals $\left(-\infty, \lambda_{0}^{\mathrm{D}}\right],\left(\lambda_{0}^{\mathrm{D}}, \lambda_{1}^{\mathrm{D}}\right], \ldots$, where the $\lambda_{n}^{\mathrm{D}}, n \geqslant 0$, are the eigenvalues for (1.1), (1.2) with the Dirichlet condition $y(1)=0$. Furthermore, $\cot \theta(1, \lambda)$ is decreasing on each branch and $\cot \theta(1, \lambda) \rightarrow \pm \infty$ as $\lambda \rightarrow \lambda_{n}^{\mathrm{D}} \pm$.

Real eigenvalues for (1.1)-(1.3) occur at $\lambda$ values for which

$$
\cot \theta(1, \lambda)=a \lambda+b
$$


A real eigenvalue $\hat{\lambda}$ is said to have algebraic multiplicity $k \geqslant 1$ if, for $l y=-y^{\prime \prime}+q y$, there is a chain of functions $y^{[0]}, \ldots, y^{[k-1]}$ with $(l-\hat{\lambda}) y^{[0]}=0,(l-\hat{\lambda})\left(y^{[j]}\right)=y^{[j-1]}$ and $y^{[j]}$ satisfying the boundary conditions (1.2) (as this boundary condition is independent of $\lambda$ ) and

$$
\left(y^{[j]}\right)^{\prime}(1)=y^{[j]}(1)(a \hat{\lambda}+b)+a y^{[j-1]}
$$

for each $1 \leqslant j \leqslant k-1$, and the chain cannot be extended to length $k+1$. Here $y^{[0]}$ is an eigenfunction for $\hat{\lambda}$ and $y^{[1]}, \ldots, y^{[k-1]}$ are the associated functions (see [15, pp. 1620] for more details). The algebraic multiplicity of an eigenvalue $\hat{\lambda}$ is $k$ if the functions $\cot \theta(1, \lambda)$ and $a \lambda+b$ and their $\lambda$ derivatives of order $1,2, \ldots, k-1$ (but not $k$ ) agree at $\hat{\lambda}$ (see [9, Lemma 2.1, Theorem 3.1] and [15, pp. 16-20]).

The following theorem on existence, multiplicity and asymptotics for eigenvalues of (1.1)-(1.3) will be a key tool in this work. From now on for simplicity we shall refer to an eigenvalue as 'belonging' to $\mathcal{B}_{k}$ if it is the abscissa of a point on $\mathcal{B}_{k}$.

Theorem 2.1. The boundary-value problem (1.1)-(1.3) has only point spectrum, which is countably infinite and accumulates at $+\infty$ and can thus be listed as $\lambda_{n}, n \geqslant 0$, with eigenvalues repeated according to algebraic multiplicity and ordered so as to have increasing real parts.

(i) For large n,

$$
\lambda_{n}= \begin{cases}\left(n-\frac{1}{2}\right)^{2} \pi^{2}+2 \cot \alpha+\frac{2}{a}+\int_{0}^{1} q+o\left(\frac{1}{n}\right), & \alpha \neq 0, \\ n^{2} \pi^{2}+\frac{2}{a}+\int_{0}^{1} q+o\left(\frac{1}{n}\right), & \alpha=0 .\end{cases}
$$

(ii) One of the following occurs:

(a) All eigenvalues are real, there are algebraically two eigenvalues on the initial branch, $\mathcal{B}_{0}$, of the Prüfer graph, and all other branches contain precisely one simple eigenvalue.

(b) All eigenvalues are real, $\mathcal{B}_{0}$ contains no eigenvalues, but, for some $k>0$, $\mathcal{B}_{k}$ contains algebraically three eigenvalues and all other branches contain precisely one simple eigenvalue.

(c) There are two non-real eigenvalues appearing as a conjugate pair, $\mathcal{B}_{0}$ contains no eigenvalues, and all other branches contain precisely one simple eigenvalue.

Proof. The first sentence follows from [16], as does the fact that all but finitely many eigenvalues are real and simple. The asymptotic development in (i) is derived in [9].

(ii) From [4, Theorem 2.4], either (a) occurs or there are no eigenvalues on $\mathcal{B}_{0}$.

In the latter case, $[\mathbf{4}$, Theorem 2.4] shows that if there are only real eigenvalues, then each branch (other than $\mathcal{B}_{0}$ ) contains at least one eigenvalue and at most one branch may contain algebraically more than one (and up to three). The asymptotics for $\lambda_{n}^{\mathrm{D}}$ are well 


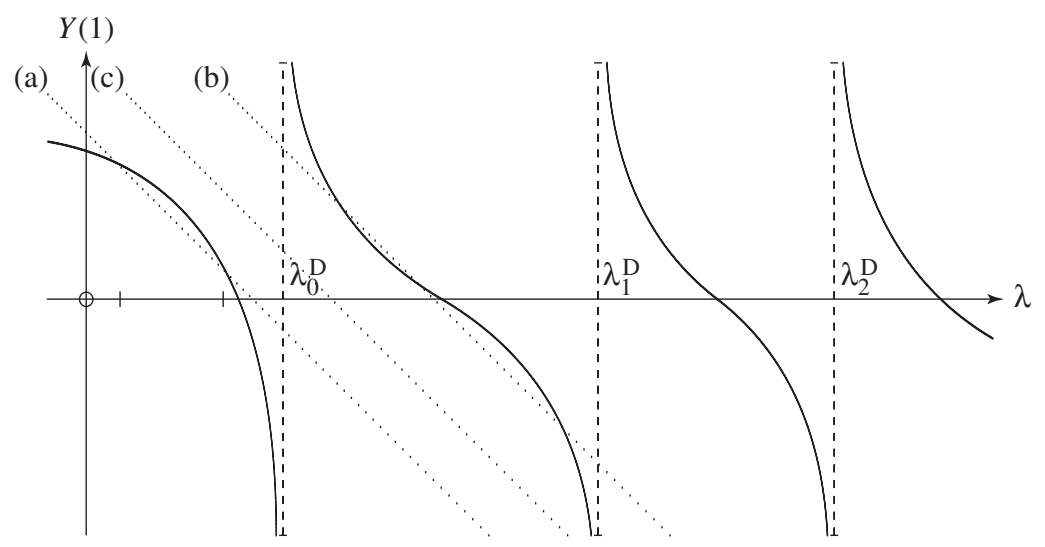

Figure 1. $\cot \theta(\lambda, 1)$.

known (cf. [5]) and are as in (i) with $n$ replaced by $n+1$. Moreover, since $a \lambda+b \rightarrow-\infty$ as $\lambda \rightarrow \infty$, we see that, for large $n, \lambda_{n+1}<\lambda_{n}^{\mathrm{D}}$. Thus in this case there will be precisely three eigenvalues on some $\mathcal{B}_{k}$ for some $k>0$, i.e. (b) holds.

Finally, in the case when complex eigenvalues are present, $[\mathbf{1 6}]$ shows that they appear in conjugate pairs, so there are at least two complex eigenvalues. Moreover, $[\mathbf{4}$, Theorem 2.4] ensures that there is exactly one real eigenvalue from each branch $\mathcal{B}_{k}$ for $k>0$. Using eigenvalue asymptotics as above, we see that there is exactly one conjugate pair of non-real eigenvalues, so (c) holds.

The above theorem is illustrated geometrically in figure 1 .

It is convenient to establish a shorthand for the cases of real eigenvalues (to be treated in $\S \S 4$ and 5) given by the above theorem. (Non-real eigenvalues, i.e. case (c) of the theorem, will be covered in $\S 6$.) The letter $D$ denotes a Dirichlet condition and $N$ signifies a non-Dirichlet condition at $x=0$. It is apparent that for cases (a) and (b) there is precisely one branch, say $\mathcal{B}_{k}$, containing algebraically more than one eigenvalue. Then we denote our problem as $D_{k}$ or $N_{k}$ depending on the boundary condition at $x=0$. These cases will be subdivided according to eigenvalue multiplicity. For example, $D_{0}(2)$ implies a double eigenvalue on $\mathcal{B}_{0}, N_{k}(2,1)$ implies a double eigenvalue followed by a simple one on $\mathcal{B}_{k}$ (necessarily $k>0$ ), and so on.

\section{Crum-type transformations}

In this section we introduce two versions of Crum's transformation and establish some of their essential properties. We begin with Crum's modification [11] of the Wronskian.

Definition 3.1. Suppose the functions $f_{1}, \ldots, f_{k}$ satisfy

$$
-f_{j}^{\prime \prime}+q f_{j}=\lambda_{j} f_{j}, \quad j=1, \ldots, k
$$


Then the 'modified' Wronskian is defined as the determinant

$$
w\left(f_{1}, \ldots, f_{k}\right)(x)=\operatorname{det}\left[f_{j}^{(i-1)}(x)\right]_{i, j=1, \ldots, k}
$$

in which $f_{j}^{(i-1)}$ is replaced by $\left(-\lambda_{j}\right)^{n} f_{j}$ if $i-1=2 n$, and by $\left(-\lambda_{j}\right)^{n} f_{j}^{\prime}$ if $i-1=2 n+1$.

To be precise, Crum used usual Wronskians for sufficiently differentiable $q$, noting that they could be replaced by the above modified versions for continuous $q$. We shall use them for $q \in L_{1}$, noting that not only $w\left(f_{1}, \ldots, f_{k}\right)(x)$, but also its first two $x$ derivatives, make sense via (1.1).

We define the Crum transformation of a solution $y$ of (1.1), with respect to the above base functions $f_{1}, \ldots, f_{k}$, by

$$
\hat{y}(x)=\frac{w\left(f_{1}, \ldots, f_{k}, y\right)(x)}{w\left(f_{1}, \ldots, f_{k}\right)(x)}
$$

where $w\left(f_{1}, \ldots, f_{k}\right)(x) \neq 0$ on $[0,1]$.

In the case $k=1$ we shall call this the Darboux transformation (cf. [14, p. 132]). Note, in the case of Sturm-Liouville boundary-value problems with eigenparameter-dependent boundary conditions of positive type (e.g. (1.3) with $a>0,(1.4)$ and in $[\mathbf{7}, \mathbf{8}]$ ), that it was enough to apply Darboux transformations with one base function, but here we shall need cases with up to four base functions.

Theorem 3.2. Let $f_{1}, f_{2}, \ldots, f_{n}, f_{n+1}$ be solutions of (1.1) with $\lambda$ taking the values $\mu_{1}, \mu_{2}, \ldots, \mu_{n}, \mu_{n+1}$, respectively. If $w\left(f_{1}, \ldots, f_{n}\right)(x) \neq 0$ for all $x \in[0,1]$, then we have the following.

(i) The function

$$
\phi=\frac{w\left(f_{1}, \ldots, f_{n}, f_{n+1}\right)}{w\left(f_{1}, \ldots, f_{n}\right)}
$$

is a solution of the equation

$$
-\phi^{\prime \prime}+\left(q-2 W\left(f_{1}, \ldots, f_{n}\right)^{\prime}\right) \phi=\mu_{n+1} \phi .
$$

(ii) If $n \geqslant 2$, then the function

$$
\psi=\frac{w\left(f_{1}, \ldots, f_{n-1}\right)}{w\left(f_{1}, \ldots, f_{n}\right)}
$$

satisfies the equation

$$
-\psi^{\prime \prime}+\left(q-2 W\left(f_{1}, \ldots, f_{n}\right)^{\prime}\right) \psi=\mu_{n} \psi .
$$

Proof. Part (i) is proved in [11], so we proceed to the proof of (ii). For convenience we write $w=w\left(f_{1}, \ldots, f_{n}\right), v=w\left(f_{1}, \ldots, f_{n-1}\right), \phi=w / v$ and $\psi=v / w$. An easy calculation shows

$$
\begin{aligned}
\phi^{\prime \prime} & =\left(\frac{v w^{\prime \prime}-2 v^{\prime} w^{\prime}-v^{\prime \prime} w}{v w}+2 V^{2}\right) \phi, \\
\psi^{\prime \prime} & =\left(\frac{w v^{\prime \prime}-2 w^{\prime} v^{\prime}-w^{\prime \prime} v}{w v}+2 W^{2}\right) \psi .
\end{aligned}
$$


From (i), we have

$$
\phi^{\prime \prime}=\left(q-2 V^{\prime}-\mu_{n}\right) \phi
$$

so (3.3) gives

$$
q-2 V^{\prime}-\mu_{n}=\frac{v w^{\prime \prime}-2 v^{\prime} w^{\prime}-v^{\prime \prime} w}{v w}+2 V^{2}
$$

whence

$$
q-\mu_{n}=\frac{v w^{\prime \prime}-2 v^{\prime} w^{\prime}+v^{\prime \prime} w}{v w}
$$

Now (3.4) gives

$$
\psi^{\prime \prime}=\left(q-\mu_{n}-\frac{2 w^{\prime \prime}}{w}+2 W^{2}\right) \psi
$$

and the result follows.

The next theorem is used to ensure the non-vanishing of the Wronskian in later situations where two base functions are used; cf. [1] for a related result.

Theorem 3.3. Let $u, z$ be solutions of (1.1), (1.2) with $\lambda$ replaced by $\mu$ and $\xi$, and $\alpha$ replaced by $\beta$ and $\gamma$, respectively. Suppose that $u$ and $z$ have the same number of zeros in $(0,1)$. If $\pi>\beta>\gamma \geqslant 0$ and $\mu>\xi$, then $w(u, z)$ is non-zero everywhere on $[0,1]$.

Proof. We can assume without loss of generality that $u$ and $z$ are normalized so that $u(0)=1, u^{\prime}(0)=\cot \beta$ and $z(0)=1, z^{\prime}(0)=\cot \gamma$ if $\gamma \neq 0, z(0)=0, z^{\prime}(0)=1$ if $\gamma=0$. First, we note that since

$$
w(u, z)(0)= \begin{cases}\cot \gamma-\cot \beta, & \gamma \neq 0 \\ 1, & \gamma=0\end{cases}
$$

we have $w(u, z)(0)>0$. Let the zeros of $u$ in $(0,1)$ be $0<a_{1}<\cdots<a_{m}<1$ and those of $z$ be $0<b_{1}<\cdots<b_{m}<1$. Sturm theory shows that $0<a_{1}<b_{1}<\cdots<a_{m}<b_{m}<1$.

Now

$$
w^{\prime}(u, z)=(\mu-\xi) u z
$$

so the critical points of $w(u, z)$ occur at $x=a_{j}, b_{j}, 1 \leqslant j \leqslant m$. From the interlacing of the $a_{j}$ and the $b_{j}$ we also see that

$$
\begin{aligned}
(-1)^{j} u^{\prime}\left(a_{j}\right)>0, & & (-1)^{j} u\left(b_{j}\right)>0 \\
(-1)^{j} z^{\prime}\left(b_{j}\right)>0, & & (-1)^{j} z\left(a_{j}\right)<0,
\end{aligned}
$$

and hence $w(u, z)>0$ at all of its critical points. Furthermore,

$$
w^{\prime \prime}(u, z)\left(b_{m}\right)=(\mu-\xi) u\left(b_{m}\right) z^{\prime}\left(b_{m}\right)>0,
$$

so the final critical point is a minimum. Thus $w(u, z)>0$ on $[0,1]$. 
The following theorem gives the analogue of Theorem 3.2 that will be used when transforming non-simple eigenvalues.

Theorem 3.4. Let $l(y)=-y^{\prime \prime}+q y$ and $y^{[j]}, j=0, \ldots, k$, be solutions to the system

$$
\begin{aligned}
& l\left(y^{[0]}\right)=\hat{\lambda} y^{[0]} \\
& l\left(y^{[j]}\right)=\hat{\lambda} y^{[j]}+y^{[j-1]}, \quad j=1, \ldots, k .
\end{aligned}
$$

Suppose that $z_{1}, \ldots z_{m}$ are solutions of (1.1) with $\lambda$ replaced by $\mu_{1}, \ldots, \mu_{m}, m \in \mathbb{N} \cup\{0\}$. If $w\left(z_{1}, \ldots, z_{m}, y^{[0]}\right)(x) \neq 0$, then the functions

$$
u^{[j-1]}=\frac{w\left(z_{1}, \ldots, z_{m}, y^{[0]}, y^{[j]}\right)}{w\left(z_{1}, \ldots, z_{m}, y^{[0]}\right)}, \quad j=1, \ldots, k,
$$

are solutions to the system (3.5), (3.6) with $k$ and $q$ replaced by $k-1$ and $q-$ $2 W\left(z_{1}, \ldots, z_{m}, y^{[0]}\right)^{\prime}$.

Proof. For each $\lambda \in \mathbb{C}$, let $g_{\lambda}$ be the solution of $(l-\lambda) g_{\lambda}=0$ with initial conditions

$$
\begin{aligned}
& g_{\lambda}(0)=\sum_{j=0}^{k}(\lambda-\hat{\lambda})^{j} y^{[j]}(0), \\
& g_{\lambda}^{\prime}(0)=\sum_{j=0}^{k}(\lambda-\hat{\lambda})^{j}\left(y^{[j]}\right)^{\prime}(0) .
\end{aligned}
$$

Straightforward computation yields

$$
y^{[j]}=\left.\frac{1}{j !} \frac{\partial^{j} g_{\lambda}}{\partial \lambda^{j}}\right|_{\lambda=\hat{\lambda}}
$$

Setting

$$
f_{\lambda}=\frac{w\left(z_{1}, \ldots, z_{m}, y^{[0]}, g_{\lambda}\right)}{w\left(z_{1}, \ldots, z_{m}, y^{[0]}\right)}
$$

we observe that $f_{\hat{\lambda}}=0$ and

$$
u^{[j-1]}=\left.\frac{1}{j !} \frac{\partial^{j} f_{\lambda}}{\partial \lambda^{j}}\right|_{\lambda=\hat{\lambda}}
$$

for $j>0$. From Theorem 3.2, we have

$$
-f_{\lambda}^{\prime \prime}+\left(q-2 W\left(z_{1}, \ldots, z_{m}, y^{[0]}\right)^{\prime}\right) f_{\lambda}=\lambda f_{\lambda},
$$

which when differentiated $j$ times with respect to $\lambda$ gives

$$
-\left(\frac{\partial^{j} f_{\lambda}}{\partial \lambda^{j}}\right)^{\prime \prime}+\left(q-2 W\left(z_{1}, \ldots, z_{m}, y^{[0]}\right)^{\prime}\right) \frac{\partial^{j} f_{\lambda}}{\partial \lambda^{j}}=\lambda \frac{\partial^{j} f_{\lambda}}{\partial \lambda^{j}}+j \frac{\partial^{j-1} f_{\lambda}}{\partial \lambda^{j-1}} .
$$

We now divide by $j$ ! and set $\lambda=\hat{\lambda}$ in the above equation to give the result. 


\section{Darboux transformations}

This section considers the simplest cases, where a (Darboux) transformation constructed from a single base function results in a Sturm-Liouville problem with constant-type boundary conditions. In these cases, the 'extra' eigenvalue noted in $\S 1$ appears on the zeroth branch $\mathcal{B}_{0}$ of the Prüfer graph. The transformation 'removes' this eigenvalue, leaving one eigenvalue per branch.

We begin with the non-Dirichlet cases (labelled $N_{0}$ at the end of $\S 2$ ), when the base functions can be taken as eigenfunctions of (1.1)-(1.3).

It is convenient to use the notation $\Lambda=\left\{\lambda_{j}: j \geqslant 0\right\}$ and

$$
\Lambda_{n}=\left\{\lambda_{j}: n \neq j \geqslant 0\right\},
$$

with the eigenvalues $\lambda_{j}$ of (1.1)-(1.3) being labelled as in Theorem 2.1.

Theorem 4.1. Let $\alpha>0$ in (1.2) and assume that $\lambda_{0} \leqslant \lambda_{1}$, both lying on the initial branch $\mathcal{B}_{0}$ of the Prüfer graph. Then the Darboux transformation with base function $y_{0}$ produces a Sturm-Liouville problem with potential

$$
\hat{q}=q-2 Y_{0}^{\prime}
$$

boundary conditions

$$
\begin{aligned}
y(0) & =0, \\
Y(1) & =-\frac{1}{a}-\left(a \lambda_{0}+b\right),
\end{aligned}
$$

and spectrum $\Lambda_{0}$.

Remark 4.2. This means, in the case of a simple eigenvalue $\lambda_{j}$ with eigenfunction $y_{j}$, that we take $f_{1}=y_{0}, y=y_{j}$ in (3.2). In the case $\lambda_{0}=\lambda_{1}$, we take $y_{0}$ as an eigenfunction for this eigenvalue and replace $y_{1}$ by the first associated function $y_{0}^{[1]}$.

Proof. Theorems 3.2 and 3.4 show that the functions

$$
u_{j}=\frac{w\left(y_{0}, y_{j}\right)}{y_{0}}
$$

(and in the case of an eigenvalue of multiplicity $2, u_{1}=w\left(y_{0}, y_{0}^{[1]}\right) / y_{0}$ ) are solutions of (1.1) with $\lambda=\lambda_{j}, j \geqslant 1$, and $q$ replaced by $\hat{q}$.

As $y_{0}$ and $y_{j}$ (and $y_{0}^{[1]}$ when considered) obey the same initial condition, which is $\lambda$ independent, it follows that $u_{j}(0)=0$.

In the case

$$
u=\frac{w\left(y_{0}, y\right)}{y_{0}},
$$

where $y$ is a solution of (1.1) obeying (1.3), we have

$$
U=W\left(y_{0}, y\right)-Y_{0}=\frac{\left(\lambda_{0}-\lambda\right) y_{0} y}{w\left(y_{0}, y\right)}-Y_{0},
$$


for $\lambda \in \mathbb{C}$. When evaluated at $x=1$ this gives

$$
U(1)=-\frac{1}{a}-\left(a \lambda_{0}+b\right)
$$

Setting $y=y_{j}$ and $\lambda=\lambda_{j}$ in the above equation we obtain the required boundary conditions at $x=1$ for the case when $\lambda_{j}$ is a simple eigenvalue.

For $\lambda_{0}=\lambda_{1}$ we take the $\lambda$ derivatives of our expression for $u$ and set $\lambda=\lambda_{0}$ giving

$$
u_{1}=\frac{w\left(y_{0},\left.\dot{y}\right|_{\lambda=\lambda_{0}}\right)}{y_{0}}=\frac{w\left(y_{0}, y_{0}^{[1]}\right)}{y_{0}} .
$$

Consequently, via the equation $-\left(y_{0}^{[1]}\right)^{\prime \prime}+q y_{0}^{[1]}=\lambda_{0} y_{0}^{[1]}+y_{0}$, we have

$$
U_{1}=W\left(y_{0}, y_{0}^{[1]}\right)-Y_{0}=-\frac{y_{0}^{2}}{w\left(y_{0}, y_{0}^{[1]}\right)}-Y_{0}
$$

but $y_{0}^{[1]}$ obeys the boundary condition

$$
\left(y_{0}^{[1]}\right)^{\prime}(1)=\left(a \lambda_{0}+b\right) y_{0}^{[1]}(1)+a y_{0}(1)
$$

and thus

$$
U_{1}(1)=-\frac{1}{a}-\left(a \lambda_{0}+b\right) .
$$

The transformed problem, which has a Dirichlet boundary condition at $x=0$ and a non-Dirichlet constant-type boundary condition at $x=1$, has eigenvalues $\mu_{0}<\mu_{1}<\cdots$, which take the asymptotic form

$$
\mu_{j}=\pi^{2}\left(j+\frac{1}{2}\right)^{2}+O(1) .
$$

In addition we have shown that each of $\lambda_{1}, \lambda_{2}, \ldots$ is an (algebraically simple) eigenvalue of the transformed problem and from Theorem 2.1,

$$
\lambda_{n}=\left(n-\frac{1}{2}\right)^{2} \pi^{2}+O(1) .
$$

Thus $\lambda_{j+1}=\mu_{j}, j=0,1, \ldots$, and $\Lambda_{0}$ constitutes the set of all eigenvalues for the transformed problem.

For Dirichlet cases we need to perturb the eigenfunctions to give the required base functions. The following theorem constructs one of the two perturbations needed in what follows.

Theorem 4.3. If all eigenvalues of (1.1)-(1.3) are real and simple, then there are at least two eigenvalues with the same oscillation count, say $n$. In particular, the secondlargest eigenvalue with oscillation count $n$ is $\lambda_{n}$ and the largest is $\lambda_{n+1}$. Then there exists $K<\cot \alpha$ such that, for each $\beta$ with $K<\cot \beta<\cot \alpha$ (where a Dirichlet condition at $x=0$ is interpreted as $\cot \alpha=+\infty)$, there exists $\mu$ with $\lambda_{n+1}>\mu>\lambda_{n}$ such that the solution $z$ of (1.1) with $\lambda=\mu$ and

$$
Z(0)=\cot \beta
$$


has oscillation count $n$ and obeys the terminal condition

$$
Z(1)=a \mu+b .
$$

Proof. The effect of decreasing $\cot \beta$ is to shift the (Prüfer) graph of $Z(1)$ to the left. We denote the $n$th branch of this graph by $\mathcal{B}_{n}(\beta)$.

If $n=0$, then $a \lambda+b$ intersects $\mathcal{B}_{n}(\alpha)=\mathcal{B}_{n}$ twice, so under sufficiently small deformation of the Prüfer graph to the left, i.e. for $\cot \beta$ in some interval of the form $(K, \cot \alpha)$, $a \lambda+b$ still intersects $\mathcal{B}_{n}(\beta)$ with abscissae $\mu_{1}(\beta)<\mu_{2}(\beta)$, say, in the interval $\left(\lambda_{n}, \lambda_{n+1}\right)$. The result follows if we take $\beta$ as above and $\mu=\mu_{1}(\beta)$.

If $n>0$, then the proof is the same except for the existence of a third intersection point with abscissa $\mu_{0}(\beta)<\mu_{1}(\beta)$.

Note 4.4. In the case $\lambda_{n-1}=\lambda_{n}<\lambda_{n+1}$ (only possible if $n \geqslant 1$ ), the above theorem is still valid.

We now consider, with the help of Theorem 4.3, the case of a Dirichlet boundary condition at $x=0$ and two simple eigenvalues on the initial branch $\mathcal{B}_{0}$ of the Prüfer graph.

Theorem 4.5. Let $\alpha=0$ in (1.2) and assume that $\lambda_{0}<\lambda_{1}$ both lie on $\mathcal{B}_{0}$. Then the Darboux transformation with base function $z$ as given in Theorem 4.3 produces a Sturm-Liouville problem with potential

$$
\hat{q}=q-2 Z^{\prime}
$$

and boundary conditions

$$
\begin{aligned}
& Y(0)=-\cot \beta=Z(0), \\
& Y(1)=-\frac{1}{a}-(a \mu+b),
\end{aligned}
$$

which is isospectral with (1.1)-(1.3).

Proof. Theorem 3.2 shows that for $\lambda=\lambda_{j}, j \geqslant 0$, and $q$ replaced by $\hat{q}$ the functions

$$
u_{j}=\frac{w\left(z, y_{j}\right)}{z}
$$

are solutions of (1.1).

As in Theorem 4.1,

$$
U_{j}=\frac{\left(\mu-\lambda_{j}\right) z y_{j}}{w\left(z, y_{j}\right)}-Z
$$

The above expression evaluated at 0 and 1 (using the boundary conditions obeyed by $z$ and $y_{j}$ ) gives

$$
U_{j}(1)=-\frac{1}{a}-(a \mu+b)
$$


and

$$
U_{j}(0)=-Z(0)
$$

Thus the $\lambda_{j}, j=0,1, \ldots$, are eigenvalues of the transformed problem.

A comparison (as for Theorem 4.1) of the asymptotic form of the eigenvalues of the transformed problem and of $\lambda_{n}$ as given in Theorem 2.1 (i) shows that $\Lambda$ constitutes the set of all eigenvalues for the transformed problem.

Remark 4.6. We have covered all the cases where (1.3) intersects $\mathcal{B}_{0}$, except for $D_{0}(2)$, in the shorthand at the end of $\S 2$.

\section{Double transformations}

In this section we discuss all remaining cases with real eigenvalues. First we show that, when all eigenvalues are real and simple, a double Crum transformation converts (1.1) (1.3) to a Sturm-Liouville boundary-value problem with real constant boundary conditions.

Theorem 5.1. Suppose that (1.1)-(1.3) has only real simple eigenvalues with $\lambda_{n-1}<$ $\lambda_{n}<\lambda_{n+1}$ on $\mathcal{B}_{n}$. Let $\beta, \mu$ and $z$ be as in Theorem 4.3. Then the Crum transformation with base functions $z$ and $y_{n}$ transforms (1.1)-(1.3) to the Sturm-Liouville problem

$$
\left.\begin{array}{c}
-u^{\prime \prime}+\left(q-2 W\left(y_{n}, z\right)^{\prime}\right) u=\lambda u, \\
U(0)=\cot \alpha+\frac{\lambda_{n}-\mu}{\cot \alpha-\cot \beta}, \quad \text { if } \alpha \neq 0, \\
u(0)=0, \quad \text { if } \alpha=0,
\end{array}\right\}
$$

with spectrum $\Lambda_{n}$ of (4.1).

Proof. Theorem 3.3 ensures that $w\left(y_{n}, z\right) \neq 0$. Throughout the proof $\lambda_{j} \in \Lambda_{n}$. Let

$$
u_{j}=\frac{w\left(y_{n}, z, y_{j}\right)}{w\left(y_{n}, z\right)}
$$

It follows from Theorem 3.2 that $u_{j}$ obeys (5.1) with $\lambda=\lambda_{j}$.

At $x=1$ we have

$$
w\left(y_{n}, z, y_{j}\right)(1)=\left|\begin{array}{ccc}
y_{n}(1) & z(1) & y_{j}(1) \\
\left(a \lambda_{n}+b\right) y_{n}(1) & (a \mu+b) z(1) & \left(a \lambda_{j}+b\right) y_{j}(1) \\
-\lambda_{n} y_{n}(1) & -\mu z(1) & -\lambda_{j} y_{j}(1)
\end{array}\right|=0
$$

which gives (5.3). 
For $\alpha=0$, let $y_{j}^{\prime}(0)=1=y_{n}^{\prime}(0)$ and $z(0)=1$. Then

$$
w\left(y_{n}, z, y_{j}\right)(0)=\left|\begin{array}{ccc}
0 & 1 & 0 \\
1 & \cot \beta & 1 \\
0 & -\mu & 0
\end{array}\right|=0
$$

and as $u_{j}^{\prime}(0) \neq 0$ it follows that the $\lambda_{j}$ are eigenvalues of (5.1)-(5.3) with eigenfunctions $u_{j}$. From $[\mathbf{9}]$

$$
\lambda_{j}=\pi^{2} j^{2}+O(1),
$$

which is the asymptotic form of the eigenvalues of (5.1)-(5.3) since $\lambda_{j} \in \Lambda_{n}$. Thus $\Lambda_{n}$ constitutes the set of eigenvalues of (5.1)-(5.3).

For $\alpha \neq 0$ assume that $y_{n}(0), y_{j}(0), z(0)=1$. Then

$$
\begin{aligned}
w\left(y_{n}, z\right)(0) & =\cot \beta-\cot \alpha, \\
w\left(y_{n}, z, y_{j}\right)(0) & =\left|\begin{array}{ccc}
1 & 1 & 1 \\
\cot \alpha & \cot \beta & \cot \alpha \\
-\lambda_{n} & -\mu & -\lambda_{j}
\end{array}\right| \\
& =\left(\lambda_{j}-\lambda_{n}\right)(\cot \alpha-\cot \beta) \neq 0, \\
w^{\prime}\left(y_{n}, z, y_{j}\right)(0) & =\left|\begin{array}{ccc}
1 & 1 & 1 \\
\cot \alpha & \cot \beta & \cot \alpha \\
-\lambda_{n} \cot \alpha & -\mu \cot \beta & -\lambda_{j} \cot \alpha
\end{array}\right| \\
& =\left(\lambda_{j}-\lambda_{n}\right)(\cot \alpha-\cot \beta) \cot \alpha .
\end{aligned}
$$

Thus $u_{j}$ is not identically zero and obeys the boundary conditions (5.2) and (5.3), showing that the $\lambda_{j}$ are eigenvalues of (5.1)-(5.3). From [9]

$$
\lambda_{j}=\pi^{2}\left(j-\frac{1}{2}\right)^{2}+O(1),
$$

which, when compared with the asymptotic form for the eigenvalues of (5.1)-(5.3), shows that $\Lambda_{n}$ is the set of all eigenvalues of (5.1)-(5.3).

For the case $\alpha>0$ there is a mirror version of Theorem 4.3 and the note thereafter. The proof is omitted as it is similar to that of Theorem 4.3.

Theorem 5.2. If $\alpha>0$, all eigenvalues of (1.1)-(1.3) are real and $\lambda_{n}=\lambda_{n+1}$ is an algebraically double eigenvalue on $\mathcal{B}_{n}$, then either $n=0$ or $\lambda_{n-1}$ is on $\mathcal{B}_{n}$ if $n \geqslant 1$. Also there exists $K>\cot \alpha$ and $\mu$ with $\lambda_{n-1}<\mu<\lambda_{n}$ (where $\lambda_{-1}$ is taken as $-\infty$ ), so that for each $\beta$ with $K>\cot \beta>\cot \alpha$, the solutions $z$ of (1.1) with $\lambda=\mu$ and

$$
Z(0)=\cot \beta
$$

have oscillation count $n$ and obey the terminal condition

$$
Z(1)=a \mu+b .
$$


Remark 5.3. Theorem 5.2 also applies to eigenvalues of multiplicity 3 , in which case $\mu>\lambda_{n+1}$.

The next result treats some cases with both single and double eigenvalues on $\mathcal{B}_{k}$ $(k>0)$, specifically $N_{k}(2,1), D_{k}(2,1)$ and $N_{k}(1,2)$ in the shorthand of $\S 2$.

Theorem 5.4. Let $\beta, \mu$ and $z$ be given by Theorem 4.3 in the case $\lambda_{n-1}=\lambda_{n}<$ $\lambda_{n+1} \in \mathcal{B}_{n}$, and by Theorem 5.2 in the case $\alpha>0, \lambda_{n-1}<\lambda_{n}=\lambda_{n+1} \in \mathcal{B}_{n}$. Then the Crum transformation of (1.1)-(1.3) with base functions $z$ and the eigenfunction $y_{n}$ for the eigenvalue $\lambda_{n}$ gives the boundary-value problem (5.1)-(5.3) with spectrum $\Lambda_{n}$ of (4.1).

Proof. Let

$$
u_{j}= \begin{cases}\frac{w\left(z, y_{n}, y_{j}\right)}{w\left(z, y_{n}\right)}, & \lambda_{j} \neq \lambda_{n}, \\ \frac{w\left(z, y_{n}, y_{n}^{[1]}\right)}{w\left(z, y_{n}\right)}, & j \neq n, \lambda_{j}=\lambda_{n}\end{cases}
$$

where $y_{n}^{[1]}$ is the first associated function at $\lambda=\lambda_{n}$ corresponding to the eigenfunction $y_{n}=y_{n}^{[0]}$. For $\lambda_{j} \neq \lambda_{n}$, that $u_{j}$ is an eigenfunction of (5.1)-(5.3) with eigenvalue $\lambda_{j}$ is proved exactly as in Theorem 5.1.

We now consider $j \neq n$ with $\lambda_{j}=\lambda_{n}$. From Theorem 3.4, $u_{j}$ is a solution of (5.1) with $\lambda=\lambda_{j}$, so it remains only to show that $u_{j}$ satisfies the boundary conditions (5.2)-(5.3).

As $\lambda_{n}$ is an eigenvalue with Jordan chain of length two we have

$$
\left(y_{n}^{[1]}\right)^{\prime}(0) \sin \alpha=y_{n}^{[1]}(0) \cos \alpha
$$

and

$$
\left(y_{n}^{[1]}\right)^{\prime}(1)=\left(a \lambda_{n}+b\right) y_{n}^{[1]}(1)+a y_{n}(1) .
$$

Thus at $x=1$ we have

$$
w\left(y_{n}, z, y_{n}^{[1]}\right)(1)=\left|\begin{array}{ccc}
y_{n}(1) & z(1) & y_{n}^{[1]}(1) \\
\left(a \lambda_{n}+b\right) y_{n}(1) & (a \mu+b) z(1) & \left(a \lambda_{n}+b\right) y_{n}^{[1]}(1)+a y_{n}(1) \\
\left(q-\lambda_{n}\right) y_{n}(1) & (q-\mu) z(1) & \left(q-\lambda_{n}\right) y_{n}^{[1]}(1)-y_{n}(1)
\end{array}\right|=0
$$

thus giving $u_{j}(1)=0$.

For $\alpha=0$, let $y_{n}$ and $z$ be normalized by $y_{n}^{\prime}(0)=1$ and $z(0)=1$ giving

$$
w\left(y_{n}, z, y_{n}^{[1]}\right)(0)=\left|\begin{array}{ccc}
0 & 1 & 0 \\
1 & \cot \beta & 0 \\
0 & -\mu & 0
\end{array}\right|=0
$$

As $u_{j}^{\prime}(0) \neq 0$, it follows that $\lambda_{j}$ is an eigenvalue of (5.1)-(5.3) with eigenfunction $u_{j}$. 
For $\alpha \neq 0$, we normalize $y_{n}$ and $z$ by $y_{n}(0)=1=z(0)$. Then

$$
\begin{aligned}
w\left(y_{n}, z, y_{n}^{[1]}\right)(0) & =\left|\begin{array}{ccc}
1 & 1 & 0 \\
\cot \alpha & \cot \beta & 0 \\
-\lambda_{n} & -\mu & -1
\end{array}\right| \\
& =\cot \alpha-\cot \beta \neq 0 \\
w^{\prime}\left(y_{n}, z, y_{n}^{[1]}\right)(0) & =\left|\begin{array}{ccc}
1 & 1 & 0 \\
\cot \alpha & \cot \beta & 0 \\
-\lambda_{n} \cot \alpha & -\mu \cot \beta & -\cot \alpha
\end{array}\right| \\
& =(\cot \alpha-\cot \beta) \cot \alpha .
\end{aligned}
$$

Thus $u_{j}$ is not identically zero and obeys the boundary conditions (5.2) and (5.3), showing that $\lambda_{j}$ is an eigenvalue of (5.1)-(5.3).

Appealing, as in Theorem 5.1, to the asymptotics for the eigenvalues of (5.1)-(5.3) and those for (1.1)-(1.3) given in Theorem 2.1, we find that $\lambda_{j}, j \neq n$, constitute the spectrum of the transformed boundary-value problem.

In the remaining cases with real spectrum, the perturbation results of Theorems 4.3 and 5.2 cannot be applied. Here, instead of using a Crum transformation to remove an eigenvalue, we use it to split double eigenvalues into two simple eigenvalues and triple eigenvalues into a double and a simple eigenvalue. The following lemma is a consequence of the fact that the asymptotes of the Prüfer graph move continuously to the left as $\cot \beta$ decreases, in the situation of Theorem 4.3 (even when there are multiple eigenvalues).

Lemma 5.5. Let $\lambda_{n-1} \leqslant \lambda_{n}=\lambda_{n+1}\left(\right.$ where $\lambda_{n-1}$ is ignored if $\left.n=0\right)$ lie on $\mathcal{B}_{n}$. Then there exists $K$ such that whenever $K<\cot \beta<\cot \alpha$ we have

$$
\lambda_{n+1}<\lambda_{n}^{\mathrm{D}}(\beta) \in \mathcal{B}_{n},
$$

where $\lambda_{n}^{\mathrm{D}}(\beta)$ denotes the $n$th eigenvalue of (1.1)-(1.3) with $\alpha$ replaced by $\beta$ and (1.3) replaced by the Dirichlet condition $y(1)=0$.

Now we are ready to discuss triple eigenvalues, and the remaining double eigenvalue Dirichlet cases, $D_{0}(2)$ and $D_{k}(1,2)$.

Theorem 5.6. If $\alpha=0$, suppose that $\lambda_{n}=\lambda_{n+1}$ is the largest eigenvalue on $\mathcal{B}_{n}$, while if $\alpha \neq 0$, suppose that $\lambda_{n-1}=\lambda_{n}=\lambda_{n+1}$. Let $\beta$ and $\lambda_{n}^{\mathrm{D}}(\beta)$ be as in Lemma 5.5 and let $z$ be a non-trivial solution of (1.1) with $\lambda=\lambda_{n}^{\mathrm{D}}(\beta)$ and

$$
Z(0)=\cot \beta \text {. }
$$

Then the Crum transformation with base functions $z$ and $y_{n}=y_{n}^{[0]}$ transforms (1.1)-(1.3) to (1.1), (1.2) with $q$ replaced by $q-2 W\left[z, y_{n}\right]^{\prime}$ and (1.3) replaced by

$$
Y(1)=a \lambda+a\left(\lambda_{n}-\lambda_{n}^{\mathrm{D}}(\beta)\right)+b .
$$

The eigenvalues of the transformed boundary-value problem are $\lambda_{j}, j \neq n$, together with $\lambda_{n}^{\mathrm{D}}(\beta)$ (which replaces $\lambda_{n}$ ). 
Proof. Theorem 3.3 ensures that $w\left(z, y_{n}\right)$ does not vanish on $[0,1]$. The proof of the theorem proceeds like the proofs of Theorems 5.1 and 5.6. Note that $\lambda_{n}^{\mathrm{D}}(\beta)=\mu$ is an eigenvalue of the transformed problem with eigenfunction

$$
e_{\mu}=\frac{y_{n}}{w\left(z, y_{n}\right)} .
$$

This follows from Theorem 3.2 and it is a routine calculation to check the boundary conditions.

The net result of the above theorem is that, while the Crum transformation has not directly given constant boundary conditions, it has produced a 'lower multiplicity' problem to which the process can be applied again, leading to constant boundary conditions via Theorems 4.5, 5.1 and 5.4. Specifically, $D_{k}(3), N_{k}(3), D_{0}(2)$ and $D_{k}(1,2)$ transform to $D_{k}(2,1), N_{k}(2,1), D_{0}(1,1)$ and $D_{k}(1,1,1)$, respectively. This follows from (5.4) and the fact that the above transformation preserves eigenvalue oscillation count. To see this, consider (1.1), (1.2) with (1.3) replaced by a Dirichlet condition. The transformation used in Theorem 5.6 is isospectral for this problem and provides the same transformed problem as given in Theorem 5.6 but with a Dirichlet condition at $x=1$. Thus the projections onto the $\lambda$-axis of the branches $\mathcal{B}_{k}$ for the original problem and for the transformed problem are identical.

\section{Complex spectrum}

The cases remaining for study involve a complex conjugate pair of non-real eigenvalues.

Lemma 6.1. Suppose that (1.1)-(1.3) has a conjugate pair of non-real eigenvalues $\lambda=\rho+\mathrm{i} \sigma$ and $\bar{\lambda}$ with eigenfunctions $f$ and $\bar{f}$. Then $f$ has no zeros in $(0,1]$.

Proof. As $f$ and $\bar{f}$ obey the same initial condition at 0 , Lagrange's formula gives

$$
w(f, \bar{f})(x)=\int_{0}^{x}\left[f \bar{f}^{\prime \prime}-f^{\prime \prime} \bar{f}\right]=2 \mathrm{i} \sigma \int_{0}^{x}|f|^{2} .
$$

Were $f\left(x_{0}\right)=0$ for some $x_{0} \in(0,1]$, this would yield $\int_{0}^{x_{0}}|f|^{2}=0$, a contradiction.

Remark 6.2. In the case $\alpha \neq 0$, we may assume $f(0)=1$ so there exist $k>c>0$ with $k \geqslant|f(x)| \geqslant c$ for all $x \in[0,1]$, i.e. we can write $f(x)=r(x) \mathrm{e}^{\mathrm{i} \Theta(x)}$, where $r$ and $\Theta$ are continuous, $\Theta(0)=0$ and $k \geqslant r(x) \geqslant c$ for all $x \in[0,1]$. In the case $\alpha=0$, we have $f(0)=0$ and we may assume that $f^{\prime}(0)=1$. Thus $f(x) / x$ has a continuous extension, $\hat{f}(x)$, to $[0,1]$ with $\hat{f}(0)=1$. Hence setting $|\hat{f}(x)|=g(x)$ we have $k_{1}>c_{1}>0$ with $c_{1} \leqslant g(x) \leqslant k_{1}$ for all $x \in[0,1]$ and a unique continuous real-valued function $\Theta$ with $\Theta(0)=0$ and $\hat{f}(x)=g(x) \mathrm{e}^{\mathrm{i} \Theta(x)}$. In particular, $f=r \mathrm{e}^{\mathrm{i} \Theta}$, where $r(x)=x g(x)$ and $r^{\prime}(0)=g(0)=1$.

Theorem 6.3. Suppose $\lambda=\rho+\mathrm{i} \sigma, \sigma>0$, is a non-real eigenvalue for (1.1)-(1.3) with eigenfunction $f(x)=r(x) \mathrm{e}^{\mathrm{i} \Theta(x)}$, where $\Theta(0)=0$ and either $r(0)=1$ if $\alpha>0$ or 
$r^{\prime}(0)=1$ if $\alpha=0$. There is $\hat{\beta} \in(0, \pi)$ such that for each $\mu$ sufficiently negative and for each $\beta_{1} \in(\hat{\beta}, \pi)$ there are $\beta_{0} \in(\alpha, \pi)$ and $z$ having no zeros in $[0,1]$, such that

$$
-z^{\prime \prime}+q z=\mu z, \quad Z(0)=\cot \beta_{0}, \quad Z(1)=\cot \beta_{1},
$$

and $R(x)>Z(x)$ for all $x \in[0,1]$ (for all $x \in(0,1]$ if $\alpha=0$ ).

Proof. We give details for the case $\alpha>0$. The adjustments needed for $\alpha=0$ are straightforward in light of Remark 6.2.

We note from Lemma 6.1 that $r(x)>0$ for all $x \in[0,1]$. With $\Phi=\Theta^{\prime}$ we have

$$
F=R+\mathrm{i} \Phi
$$

and so $R(0)=\cot \alpha, \Phi(0)=0$.

Now (1.1) is

$$
-\left(r \mathrm{e}^{\mathrm{i} \Theta}\right)^{\prime \prime}+q r \mathrm{e}^{\mathrm{i} \Theta}=(\rho+\mathrm{i} \sigma) r \mathrm{e}^{\mathrm{i} \Theta},
$$

and by equating real and imaginary parts, we have

$$
\begin{aligned}
-r^{\prime \prime}+\left(q+\Phi^{2}\right) r & =\rho r, \\
r \Phi^{\prime}+2 r^{\prime} \Phi+\sigma r & =0 .
\end{aligned}
$$

From (6.1) and the equation

$$
w(f, \bar{f})=-2 \mathrm{i} \Theta^{\prime} r^{2}
$$

we obtain

$$
\Phi(x)=-\frac{\sigma}{r^{2}(x)} \int_{0}^{x} r^{2}(s) \mathrm{d} s .
$$

This, along with Remark 6.2 , shows that $\Phi$ has a continuous extension to $[0,1]$, negative on $(0,1]$, with $\Phi(0)=0$. Hence there is a constant $\kappa>0$ such that $-\kappa \leqslant \Phi(x) \leqslant 0$ for all $x \in[0,1]$.

We now consider (6.2) as a Sturm-Liouville equation with initial condition $\left(r^{\prime} / r\right)(0)=$ $\cot \alpha$. The corresponding Prüfer angle $\theta_{r}$, say, satisfies

$$
\theta_{r}^{\prime}(x)=\cos ^{2} \theta_{r}(x)+\left(\rho-q(x)-\Phi^{2}(x)\right) \sin ^{2} \theta_{r}(x), \quad \theta_{r}(0)=\alpha,
$$

and since $r \neq 0$ on $[0,1]$, it follows that $0<\theta_{r}(1)<\pi$. Select $\beta_{1} \in\left(\theta_{r}(1), \pi\right)$ and let $\hat{\mu}>\kappa^{2}$ be arbitrary. The Prüfer equation

$$
\theta^{\prime}=\cos ^{2} \theta+(\rho-q-\hat{\mu}) \sin ^{2} \theta, \quad \theta(1)=\beta_{1},
$$

has a unique solution $\hat{\theta}$, say, and Sturm's Comparison Theorem shows that $\hat{\theta}(x)>\theta_{r}(x)$ for all $x \in[0,1]$. Thus $\hat{\theta}(0)>\alpha$ and, moreover, $\hat{\theta}(0)<\pi$ since $\hat{\theta}$ can only increase through multiples of $\pi$. We take $\beta_{0}=\hat{\theta}(0)$. The upshot is that there is a function $z$ on $[0,1]$ for which $Z(x)=\cot \hat{\theta}(x)$, satisfying the demands of the lemma with $\mu=\rho-\hat{\mu}$. 


\subsection{The non-Dirichlet case $(\alpha>0)$}

We list the spectrum as $\lambda_{0}, \bar{\lambda}_{0}, \lambda_{1}, \lambda_{2}, \ldots$ with corresponding eigenfunctions $f, \bar{f}$, $y_{1}, y_{2}, \ldots$ We construct $z$ as in Lemma 6.3 and calculate

$$
w(z, f, \bar{f})=-\left|\begin{array}{ccc}
z & f & \bar{f} \\
z^{\prime} & f^{\prime} & \bar{f}^{\prime} \\
\mu z & \lambda_{0} f & \bar{\lambda}_{0} \bar{f}
\end{array}\right|,
$$

which after some manipulation simplifies to

$$
2 \mathrm{i} z|f|^{2}((\mu-\rho) \Phi+\sigma(R-Z)) .
$$

Here, as above, $\lambda_{0}=\rho+\mathrm{i} \sigma, \sigma>0$. Lemma 6.3 now shows that $w(z, f, \bar{f})$ does not vanish on $[0,1]$.

This leads us to a Crum transformation with three base functions $z, f$ and $\bar{f}$ generating a new potential $\hat{q}=q-2 W(z, f, \bar{f})^{\prime}$. The eigenfunctions

$$
e_{n}=\frac{w\left(z, f, \bar{f}, y_{n}\right)}{w(z, f, \bar{f})}, \quad n \geqslant 1,
$$

for eigenvalues $\lambda_{n}, n \geqslant 1$, satisfy

$$
e_{n}(0)=0, \quad E_{n}(1)=-\frac{1}{a}-W(z, f, \bar{f})(1)
$$

These calculations are easily performed. It is important to note that $\mu$ is also an eigenvalue for the transformed problem with eigenfunction

$$
e_{\mu}=\frac{w(f, \bar{f})}{w(z, f, \bar{f})} .
$$

Indeed, Theorem 3.2 verifies that $e_{\mu}$ obeys the transformed differential equation, while tedious but routine calculations give the new boundary conditions.

We can summarize this discussion with the following theorem.

Theorem 6.4. If (1.1)-(1.3) has $\alpha>0$ and non-real eigenvalues, then there is a Crum transformation with three base functions (two of which are eigenfunctions for the conjugate pair of non-real eigenvalues) transforming (1.1)-(1.3) to a problem with Dirichlet condition at $x=0$ and non-Dirichlet (constant) boundary condition at $x=1$ and the same spectrum as (1.1)-(1.3), but with the non-real eigenvalues replaced by one real eigenvalue $\mu$ below the least real eigenvalue of the initial problem.

The net result is that the transformed problem has constant boundary conditions, the initial condition being Dirichlet, and spectrum $\mu, \lambda_{1}, \lambda_{2}, \ldots$ 


\subsection{The Dirichlet case}

Modifications must be made to the above method when $\alpha=0$. We select $\mu$ large and negative and construct $z$ as before (along with $\beta_{0}$ and $\beta_{1}$ ). Then with $\nu<\mu$ and $\tilde{\beta}_{1}>\beta_{1}$, we repeat the construction to obtain another non-vanishing function $v$ with

$$
-v^{\prime \prime}+q v=\nu v, \quad V(0)=\tilde{\beta}_{0}, \quad V(1)=\tilde{\beta}_{1}, \quad \tilde{\beta}_{1}>\beta_{1}, \quad \tilde{\beta}_{0}>\beta_{0},
$$

and, evidently,

$$
R(x)>Z(x)>V(x), \quad x \in(0,1] .
$$

Then we can verify

$$
w(v, z, f, \bar{f})(0)=2 \mathrm{i} \sigma v(0) z(0)\left|f^{\prime}(0)\right|^{2}(\mu-\nu)
$$

and

$$
\begin{aligned}
\frac{w(v, z, f, \bar{f})}{2 \mathrm{i} v z|f|^{2}}=(V-Z) \Phi \sigma^{2}+(\mu-\nu) \sigma \Phi^{2} \\
+(V-Z)(\rho-\nu)(\rho-\mu) \Phi+(\mu-\nu)(R-V)(R-Z) \sigma
\end{aligned}
$$

on $(0,1]$. Since $\Phi<0$ on $(0,1]$ and $\rho>\mu>\nu$, we see that $w(v, z, f, \bar{f})$ does not vanish on $[0,1]$.

This leads to the use of a Crum transformation with the four base functions $v, z, f, \bar{f}$ producing a new problem with eigenvalues $\nu<\mu<\lambda_{1}<\lambda_{2}<\cdots$, eigenfunctions

$$
e_{\nu}=\frac{w(z, f, \bar{f})}{w(v, z, f, \bar{f})}, \quad e_{\mu}=\frac{w(v, f, \bar{f})}{w(v, z, f, \bar{f})}, \quad e_{n}=\frac{w\left(v, z, f, \bar{f}, y_{n}\right)}{w(v, z, f, \bar{f})}, \quad n \geqslant 1,
$$

and boundary conditions

$$
y(0)=0, \quad Y(1)=c \lambda+d,
$$

for some real constants $c$ and $d$. As before, Theorem 3.2 verifies that $e_{\nu}, e_{\mu}$ and $e_{n}$ are solutions of the transformed differential equation, and the boundary conditions (6.4) follow from a routine, but tedious, calculation.

In brief, we have deduced the following theorem.

Theorem 6.5. If (1.1)-(1.3) has $\alpha=0$ and non-real eigenvalues, then there is a Crum transformation with four base functions (two of which are eigenfunctions for the conjugate pair of non-real eigenvalues) that transforms (1.1)-(1.3) to a problem with boundary conditions (6.4) and with the same spectrum as (1.1)-(1.3), but with the nonreal eigenvalues replaced by two (distinct) real eigenvalues below the least real eigenvalue of the initial problem.

Finally, we note that although $c<0$ (since the new eigenfunctions $e_{\nu}, e_{\mu}$ both have no internal zeros in $(0,1))$, the transformed problem is of type $D_{0}(1,1)$, to which Theorem 4.5 can be applied. 
Acknowledgements. The research of P.A.B. was supported by the I. W. Killam Foundation and the NSERC of Canada. The research of P.J.B. was supported by the NSERC of Canada. The research of B.A.W. was conducted while visiting the University of Calgary and the University of Saskatchewan and supported in part by the Centre for Applicable Analysis and Number Theory.

\section{References}

1. V. É. AdLER, A modification of Crum's method, Theoret. Math. Phys. 101 (1994), 13811386 .

2. F. V. AtKinson, Discrete and continuous boundary value problems (Academic, 1964).

3. A. I. Benedek and R. Panzone, On Sturm-Liouville problems with the square-root of the eigenvalue parameter contained in the boundary condition, Notas Alg. Analisis 10 (1981), 1-59.

4. P. A. Binding And P. J. Browne, Applications of two parameter eigencurves to SturmLiouville problems with eigenparameter-dependent boundary conditions, Proc. R. Soc. Edinb. A 125 (1995), 1205-1218.

5. P. A. Binding, P. J. Browne and K. Seddighi, Sturm-Liouville problems with eigenparameter dependent boundary conditions, Proc. Edinb. Math. Soc. 37 (1993), 57-72.

6. P. A. Binding, P. J. Browne and B. A. Watson, Transformations between SturmLiouville problems with eigenvalue dependent and independent boundary conditions, Bull. Lond. Math. Soc. 33 (2001), 749-757.

7. P. A. Binding, P. J. Browne and B. A. Watson, Sturm-Liouville problems with boundary conditions rationally dependent on the eigenparameter, I, Proc. Edinb. Math. Soc. 45 (2002), 631-645.

8. P. A. Binding, P. J. Browne and B. A. Watson, Sturm-Liouville problems with boundary conditions rationally dependent on the eigenparameter, II, Proc. Edinb. Math. Soc. 148 (2002), 147-168.

9. P. A. Binding, P. J. Browne And B. A. Watson, Equivalence of inverse SturmLiouville problems with boundary conditions rationally dependent on the eigenparameter, J. Math. Analysis Applic. 291 (2004), 246-261.

10. L. Collatz, Eigenwertaufgaben mit technischen Anwendungen (Akademische, Leipzig, 1963).

11. M. M. Crum, Associated Sturm-Liouville systems, Q. J. Math. (2) 6 (1955), 121-127.

12. A. DiJKSMA AND H. LANGER, Operator theory and ordinary differential operators, Fields Inst. Monographs 3 (1996), 75-139.

13. C. T. FUlton, Two-point boundary value problems with eigenparameter contained in the boundary conditions, Proc. R. Soc. Edinb. A 77 (1977), 293-308.

14. E. L. INCE, Ordinary differential equations (Dover, 1956).

15. M. A. NAimark, Linear differential operators, vol. I (Frederick Ungar, New York, 1967).

16. E. M. RUSSAKOVSKII, Operator treatment of boundary problems with spectral parameters entering via polynomials in the boundary conditions, Funct. Analysis Applic. 9 (1975), 358-359.

17. A. V. Štraus, On spectral functions of differential operators, Izv. Akad. Nauk SSSR Ser. Mat. 19 (1955), 201-220.

18. J. WALteR, Regular eigenvalue problems with eigenvalue parameter in the boundary conditions, Math. Z. 133 (1973), 301-312. 